\title{
FINASTERIDA E CALVÍCIE ANDROGENÉTICA: em busca de uma terapêutica embasada na atenção integral à saúde do homem
}

Bruno Ferreira F. LAIGNIER ${ }^{1}$

Ellen Unoki FAJARDO²

Lara Élida Guazzeli de FREITAS ${ }^{3}$

Maria Luiza Pereira NUNES ${ }^{4}$

Samuel Nuno Pereira LIMA ${ }^{5}$

Carla Rosane Ouriques COUTO ${ }^{6}$

Gérsika Bitencourt SANTOS ${ }^{7}$

\begin{abstract}
${ }^{1}$ Acadêmico do curso de Ciências Médicas da Universidade José do Rosário Vellano, Alfenas - MG, email:brunoffl@ hotmail.com;

${ }^{2}$ Acadêmica do Curso de Ciências Médicas da Universidade José do Rosário Vellano, Alfenas - MG, e-mail: ellen_unoki@hotmail.com;

${ }^{3}$ Acadêmico do curso de Ciências Médicas da Universidade José do Rosário Vellano, Alfenas - MG, e-mail: lara_freitas23@hotmail.com;

${ }^{4}$ Acadêmico do curso de Ciências Médicas da Universidade José do Rosário Vellano, Alfenas - MG, e-mail: marialuizapnunes@hotmail.com;

${ }^{5}$ Acadêmico do curso de Medicina da Universidade Federal do Amazonas, Manaus-AM, e-mail: samuelnplima@yahoo.com.br;

${ }^{6}$ Mestre em pediatria, saúde pública, saúde do trabalhador, gerenciamento de unidades básicas de saúde e medicina da família e comunidade, mestre em psicologia social, terapeuta familiar e de grupos, docente da Universidade José do Rosário Vellano, Alfenas - MG, e-mail:karllakouto@ hotmail.com.

${ }^{7}$ Doutoranda em Ciências Farmacêuticas pela Universidade Federal de Alfenas, Mestre em Ciências farmacêuticas pela Universidade Federal de Alfenas, Professora de Farmacologia do curso de Ciências Médicas da Universidade José do Rosário Vellano, Alfenas - MG, e-mail: gersika.santos@unifenas.br.
\end{abstract}

Recebido em: 28/07/2016 - Aprovado em: 28/03/2017 - Disponibilizado em: 01/07/2017

\section{RESUMO}

A perda de cabelo e calvície são afecções comuns, que devido à mudança no padrão de comportamento do gênero masculino e aos importantes efeitos psicológicos e econômicos, têm levado ao aumento da procura por tratamento dessas condições. Um dos tipos mais comuns de calvície é a alopéciaandrogenética, cujos mecanismos fisiopatológicos ainda não estão completamente elucidados, porém, encontram-se diretamente associados a fatores hereditários e a dinâmica da produção de androgênios. Por tratar-se de uma condição muito prevalente na população masculina, e terapêutica baseada na prescrição de baixa dosagem de finasterida tem se tornado comum nos consultórios médicos. Os benefícios e riscos do uso da finasterida para o tratamento de queda de cabelo têm sido recentemente centro de importantes discussões tanto na literatura técnico-científica, quanto da grande mídia, especialmente devido ao possível potencial de disfunção sexual irreversível. Grandes estudos divergem quanto aos impactos do uso da finasterida sobre a 
saúde masculina. Esta revisão bibliográfica realiza a proposta de analisar o tema sobre a ótica da atenção integral à saúde do homem, debatendo temas associados como, interação entre o paciente e o serviço de saúde, ansiedade, depressão, libido, disfunção erétil, infertilidade.

DESCRITORES: Alopécia. Finasterida. Saúde do Homem. Função erétil. Qualidade de Vida.

\title{
FINASTERIDE AND ANDROGENETIC BALDNESS: in search of a therapeutic guided by the integral attention to men's health
}

\begin{abstract}
Hair loss and baldness are common diseases which due to the change in the male gender behavior pattern and the important psychological and economic effects have led to the increased demand for treatment of these conditions. One of the most common types of hair loss is androgenetic alopecia which pathophysiological mechanisms are not fully elucidated yet, however, are directly linked to hereditary factors and the dynamics of production of androgen. For being a very prevalent condition in the male population and therapeutic based on prescription of low dosage of finasteride it has become common in doctors' offices.

The benefits and risks of finasteride usage for the hair loss treatment have recently been the center of important discussions both in technical and scientific literature, as the mainstream media, especially because of the possible potential for permanent sexual dysfunction. Large studies differ as to the impacts of finasteride usage on men's health. This bibliographic review achieves the proposal of analyse the topic on the perspective of the integral attention to men's health care, debating issues associated such as interaction between the patient and the health service, anxiety, depression, libido, erectile dysfunction, infertility.
\end{abstract}

DESCRIPTORS: Alopecia. Finasteride.Men's Health.Erectile function.Quality of life.

\section{INTRODUÇÃO}

A recente criação pelo Ministério da Saúde Brasileiro de uma Política Nacional de Atenção Integral à Saúde do Homem, do ponto de vista histórico das políticas de saúde voltadas a populações específicas, é um momento significativo no longo e contraditório processo em torno da medicalização do homem. Embora ainda encontre-se em fase inicial, e receba pouca ênfase quando comparada a outras demandas em saúde pública, a implementação de ações direcionadas para a atenção integral a saúde do homem visa quebrar estereótipos de gênero, ao mesmo tempo em que objetiva compreender a masculinidade à luz das principais determinantes sociais de saúde. Em suma categórica, toda oportunidade de se abordar um homem, especialmente na atenção primária, consiste em um importante momento para promover intervenções que levem em consideração aspectos além da biologia individual,como estilo de vida, necessidade de acesso a outros serviços de saúde, e os contextosambientais, culturaise psicossociais.

No início da primeira década do século XXI, o processo de objetificação dos homens e de seus corpos ganhou um novo ritmo. A transformação das estruturas familiares e de padrões de masculinidade tem permitido aos homens emergirem como consumidores de bens e serviços, entre eles os serviços de saúde, antes voltados às mulheres 
ou vistos como intrinsecamente femininos (CARRARAet al. 2009).

A perda de cabelo e calvície são os problemas mais comuns da sociedade moderna e, devido a essa mudança no padrão de preocupação do homem, têm levado ao aumento da procura por tratamento dessas condições, que criam muitos efeitos econômicos e psicológicos. Um dos tipos mais comuns de alopecia é a calvície ou alopécia androgenética. Recentemente, um grande esforço tem sido feito para tratar a perda de cabelo resultando em diferentes estratégias terapêuticas com resultados clínicos variados e diferentes níveis de acessibilidade (HAJHEYDARIet al. 2009).

A alopéciaandrogenética (AAG) é uma condição dermatológica crônica, também conhecida como calvície de padrão masculino, presente em aproximadamente $30 \%$ dos homens brancos na idade de 30 anos, pelo menos $50 \%$ na idade de 50 anos e $80 \%$ na idade de 70 anos. É caracterizada pelo progressivo estreitamento de cabelo na área do vértice e fronto-temporal do couro cabeludo em pessoas predispostas geneticamente. Nesta patologia observa-se um encurtamento da fase anágena do ciclo do pelo, que se caracteriza por intensa atividade mitótica na matriz do folículo piloso; e prolongamento da fase telógena, em que o pelo se separa da papila dérmica. Portanto, as hastes dos pelos caem muito antes do início de uma nova fase anágena, ocorrendo um período de latência em que não há pelo no canal folicular (STOUGHet al. 2005).

A fase evolutiva da AAG na qual o indivíduo é diagnosticado possui forte influência sobre o tipo de tratamento a ser disponibilizado. De um modo geral, os benefícios do tratamento farmacológico mostram-se superiores para os pacientes que apresentem os primeiros sinais de calvície de padrão masculino, ao passo que aqueles que apresentam manifestações avançadas da patologia pouco se beneficiam do mesmo, estando sujeitos a recorrer a outras estratégias, como o uso de próteses capilares ou a execução de transplante capilar.

Os mecanismos fisiopatológicos responsáveis pelas alterações na alopécia androgenética ainda não estão completamente elucidados. Porém, os androgênios em associação com os fatores hereditários estão envolvidos na patogênese (LORETOet al. 2014). Foi observado no couro cabeludo calvo níveis excessivos da enzima $5 \alpha$-redutase, diidrotestosterona e receptor de androgênio. O mecanismo exato por meio do qual o androgênio age parece estar relacionado à expressão dos genes que controlam os ciclos foliculares ${ }^{5}$. Além disso, um estudo tem demostrado que níveis elevados de diidrotestosterona têm sido relacionados com indução de apoptose em células de papila dérmica(WINIARSKA et al. 2006). Juntas, essas características biológicas 
fundamentaram propostas farmacológicas que tivessem como alvo molecular a inibição da enzima $5 \alpha$-redutase e a consequente diminuição dos efeitos deletérios associados à elevada produção de diidrotestosterona nos folículos capilares, dentre as quais o emprego da finasterida demonstrou-se viável e tornando-se comercialmente disponível.

\section{A testosterona e diidrotestosterona} (DHT) desempenham papéis críticos no desenvolvimento e crescimento normais dos sistemas reprodutivos e não reprodutivos masculinos (LEE, CHANG, 2003). A enzima $5 \alpha$-redutase converte a testosterona em DHT e existe em duas formas de isoenzimas: tipo I, predominante no fígado e tipo II, predominante na próstata, vesículas seminais e folículos pilosos. Em todos esses locais a testosterona é convertida em DHT. As suas ações biológicas são mediadas pelo receptor de androgênio (AR), um fator de transcrição dependente de ligante, que pertencem à superfamília de receptores nucleares. O complexo androgênio-AR interage com vários fatores para modular a transcrição de genesalvo via sequências específicas de DNA (MYSORE, 2012). A isoenzima do tipo II é encontrada na bainha externa da raiz dos folículos pilosos e papilas dérmicas e tem papel mais importante no padrão de queda do cabelo. $\mathrm{O}$ envolvimento dos androgênios na AAG é evidente, uma vez que os pseudohermafroditas masculinos possuem uma mutação no gene que codifica a $5 \alpha$-redutase II, o que prejudica a conversão da testosterona em DHT. Esses indivíduos não desenvolvem alopéciaandrogenética e nem hiperplasia prostática (LEE, CHANG, 2003). Como consequência desse fato, é natural que se a elenque a hipótese de que os efeitos do uso de inibidores de $5 \alpha$-redutase sobre o organismo masculino estendam-se além do folículo capilar, correlacionando-se efeitos em outros órgãos e repercussões neuro-imunoendócrinas.

Embora a alopécia seja vista como uma condição dermatológica relativamente suave, é uma condição física e psicologicamente prejudicial, visto que o cabelo tornou-se um componente importante na identidade e na auto-estima. A finasterida encontra-se entre os principais fármacos utilizados.

Diante da relevância do tema, este trabalho propõem-se a discutir o papel da finasterida, seus principais efeitos adversos e suas repercussões sobre a saúde masculina, buscando-se compreender todas as nuances relacionadas à prescrição do fármaco e buscando que tal ato seja guiado por uma visão holística e direcionado para a atenção integral a todas às repercussões que o mesmo possa ter sobre a saúde do homem. 


\section{MÉTODOS}

Foi realizada uma revisão narrativa da literatura por meio de uma busca bibliográfica nas seguintes bases de pesquisa online:Pubmed, BIREME, Scielo e Sciencedirect, limitando-se a busca ao período de 2000 a 2016. Os artigos obtidos através das estratégias de busca, que tiveram como temática principal “disfunção erétil em pacientes que usam finasterida", foram avaliados e classificados em elegíveis (estudos que apresentaram relevância clínica e tinham possibilidade de serem incluídos na revisão) e não elegíveis (estudos sem relevância, sem possibilidade de inclusão na revisão). Dentre os critérios observados para a escolha dos artigos foram considerados os seguintes aspectos: disponibilidade do texto integral do estudo e clareza no detalhamento metodológico utilizado.

\section{RESULTADOS E DISCUSSÃO}

A finasterida é ainda uma das drogas terapêuticas mais comumente prescritas para alopéciaandrogenética. É um inibidor da enzima $5 \alpha$-redutase II e foi desenvolvida inicialmente para o tratamento de pacientes com sintomas de hiperplasia prostática benigna. Posteriormente, a finasterida foi também aprovada para o tratamento de AAG (TRAISHet al, 2011).
A eficácia clínica da finasterida foi documentada em ensaios clínicos controlados que monitoraram os homens com calvície de padrão masculino. Em comparação com placebo, a finasterida diminuiu perda e aumentou a densidade e comprimento do cabelo, efeitos que foram mantidos durante todo o tratamento (WHITING et al, 2003). Estas melhorias foram associadas com o aumento da satisfação do paciente e da sua imagem corporal. A maioria dos pacientes relatou que estavam satisfeitos com a aparência geral de seu couro cabeludo no final de cinco anos de tratamento (KAUFMAN, 2002).

Uma recente e relevante metanálise analisou a notificação de eventos adversos em ensaios clínicos de finasterida para alopéciaandrogenética. Dos 34 ensaios clínicos, nenhum apresentou relatórios de segurança adequados, 19 foram parcialmente adequados, 12 eram insuficientes e três não relataram efeitos adversos (BELKNAPet al, 2015). Depreende-se portanto, que as conclusões e relevância das informações disponíveis nos ensaios clínicos sobre uso da finasterida em homens com AAG são muito limitadas, de má qualidade e sistematicamente tendenciosa, sendo insuficientes para estabelecer o perfil de segurança da finasterida.

$$
\text { O uso de finasterida para o tratamento }
$$
de queda de cabelo tem sido recentemente o 
foco de atenção da mídia devido ao possível potencial de disfunção sexual irreversível e desenvolvimento de depressão (SINGH, AVRAM, 2014). No entanto, grandes estudos divergem quanto à possibilidade de disfunção sexual causada pelo uso de finasterida. Nos próximos tópicos nos deteremos na análise dos aspectos universais relacionados à prescrição do fármaco, ressaltando bases biológicas e correlações clínicas em diferentes graus de evidência científica.

\section{ATENDIMENTO INICIAL AO HOMEM COM QUEIXA DE QUEDA CAPILAR}

O momento em que um homem procura o serviço de saúde, independente da queixa principal apresentada pelo mesmo, sempre consistirá em situação oportuna para realizar um atendimento baseado na política nacional de atenção integral à saúde do homem. Mesmo diante de uma queixa aparentemente vinculada a aspectos estéticos, como a queda capilar, faz-se necessário realizar ampla abordagem do paciente através de anamnese completa e exame físico, bem como o rigoroso exercício do diagnóstico diferencial.

O contato inicial com o paciente poderá revelar importantes condições clínicas subjacentes, as quais poderiam não ser identificadas e manejadas caso a oportunidade fosse desperdiçada. Sendo assim, deve-se estar atento a informações relacionadas ao estilo de vida, ao histórico patológico pregresso e, principalmente, aos sinais e sintomas associados. A identificação de fatores como estresse crônico, tabagismo, etilismo, sedentarismo e comportamento social ou sexual de risco, podem contribuir para a identificação de doenças cardiovasculares, metabólicas, nutricionais, reprodutivas e psiquiátricas.

Como já mencionado, o uso continuo da finasterida tem sido correlacionado na literatura, ainda que em meio a controvérsias, principalmente com a ocorrência de ansiedade, depressão, disfunção erétil, perda de libido e redução da fertilidade, logo, faz-se importante identificar se essas condições já se encontram presentes no momento do diagnóstico de AAG e em estágio anterior à prescrição do fármaco.

\section{SISTEMA NEURO-ENDÓCRINO E POSSÍVEIS EFEITOS ADVERSOS DA FINASTERIDA}

Estudos realizados em modelos animais tem evoluído no sentido de demonstrar que esteroides sexuais podem desempenhar importante papel na modulação de funções relacionadas ao sistema nervoso central, já tendo sido demonstrada a atuação de esteróides sobre o sistema límbico, sugerindo mecanismos regulatórios capazes de influenciar até mesmo o desempenho de funções executivas. Seguindo tal raciocínio, ao se utilizar de uma estratégia terapêutica 
capaz de afetar a geração ou biodisponibilidade de um esteroide sexual, além dos efeitos classicamente relacionados ao aumento ou redução de sua concentração, outros eventos adversos, por vezes, até mesmo mais subjetivos, poderiam ser resultado da modulaçãode suas ações endócrinas, parácrinas e autócrinas. A literatura aponta para a forte associação existente entre androgênios, neurogênesehipocampal e possíveis prejuízos ao humor e capacidade cognitiva quando vias de sinalização mediadas por androgênios são interrompidas (MAHMOUD et al., 2016). O estudo realizado por Romer e colaboradores,2010, demonstrou que o uso de finasterida correlacionou-se com redução de neurogênese no hipocampo de roedores. Obviamente, as condições experimentais empregadas em modelos animais podem ser difíceis de se extrapolar para humanos, no entanto revelam a possibilidade de existência de mecanismos farmacológicos semelhantes capazes de embasar efeitos adversos. Considerando-se a influência do sistema límbico sobre a libido e o comportamento sexual, também seria possível aventar a possibilidade de que, além de possuir efeito negativo sobre o humor em indivíduos susceptíveis, os mecanismos citados possuam alguma contribuição na gênese eventos adversos como perda de libido e disfunção erétil relacionados ao uso da finasterida.

\section{FINASTERIDA,}

ANSIEDADE

$\mathbf{E}$

\section{DEPRESSÃO}

Efeitos colaterais como ansiedade e depressão podem ser evidentes e persistentes com o uso da finasterida. Uma hipótese possível para explicar os sintomas de depressão após o tratamento com finasterida é a insuficiência nos níveis de esteroides neuroativos. Um estudo avaliou os níveis de esteroides neuroativos no plasma e amostras do líquido cefalorraquidiano (LCR), por espectrometria de massa, obtidos de pacientes do sexo masculino após a descontinuação da droga. Notou-se diminuição dos níveis de tetraidroprogesterona, isopregnenolona e de diidrotestosterona no LCR dos pacientes pós uso de finasterida em comparação com o grupo controle, evidenciando, portanto, que uma deficiência dos níveis de esteroides neuroativos, associada a sintomas de depressão, ainda está presente em pacientes com alopéciaandrogenética tratados com finasterida em baixa dose, apesar da descontinuação do tratamento (MELCANGIet al., 2013). Esses efeitos persistentes foram reconhecidos como uma possível "síndrome pós-finasterida" (GANZER et al., 2014).

\section{FINASTERIDA E FUNÇÃO SEXUAL}

A causa específica da provável disfunção sexual pelo uso da finasterida ainda não está completamente elucidada. Entretanto, vários 
estudos têm demonstrado que os androgênios são essenciais para manter a integridade estrutural da dorsal peniana e nervos cavernosos, do músculo liso e do tecido conjuntivo do corpo cavernoso e da via de sinalização no pênis. Assim, a deficiência de androgênio induzida por inibição da $5 \alpha$ redutase pode contribuir para disfunção erétil (TRAISH et al., 2011). Em um ensaio clínico randomizado, duplo-cego, utilizando 120 homens com idades entre 50 e 70 anos, foi prescrito diidrotestosterona na forma de gel transdérmico diário. Notou-se melhora no índice internacional de função erétil, sugerindo que o tratamento com DHT mantém ereção de 3 a 6 meses (KUNELIUSet al., 2013).

Segundo estudo comparativo, o perfil de reações adversas do finasterida é equivalente ao placebo, exceto para os efeitos sexuais, que foram relatados ligeiramente mais frequentes com uso de finasterida (diminuição da libido $1,3 \%$ vs. $1,8 \%$, disfunção erétil $0,7 \%$ vs. $1,3 \%$ e distúrbios ejaculatórios $0,7 \%$ vs. $1,2 \%$ ). Os efeitos sexuais muitas vezes diminuem ou desaparecem com a continuação do tratamento e revertem com a interrupção (PRICEet al., 1999).

Por outro lado, recentemente, tem sido relatado que os efeitos adversos da finasterida, além de prevalentes, podem ser persistentes por vários meses ou anos após a interrupção do medicamento. Em 2011, um estudo realizado por Irwige eKolukula, caracterizou os tipos e duração dos efeitos colaterais sexuais persistentes que ocorreram em 71 homens saudáveis durante ou imediatamente após tomar finasterida para o tratamento da AAG. A maioria dos homens desenvolveu disfunção sexual em vários domínios, com 94\% apresentando baixa libido, 92\% experimentando disfunção erétil, $92 \%$ diminuição da excitação e $69 \%$ relatando problemas com orgasmo (IRWIG, KOLUKULA, 2011).Evidências adicionais são encontradas em estudos clínicos que sugerem fortemente que, em alguns pacientes, os efeitos adversos sexuais são persistentes (MOTOFEIet al., 2013).

Um pequeno estudo investigou as razões de sensibilidade reduzida ou alteradas na área genital em oito homens que tomaram finasterida e que também sofriam de disfunção sexual, incluindo desconforto, dormência ou mesmo parestesia da pele na região genital, perda persistente da libido e disfunção erétil mais de seis meses após a interrupção do tratamento. Todos os pacientes declararam que o uso de finasterida efetivamente cessou a perda de cabelo. Porém, todos alegaram persistência dos efeitos colaterais sexuais, indicando disfunção sexual com perda da sensibilidade do pênis, diminuição da resposta agradável ao toque, flacidez e retração do escroto. As reais causas e incidência desses fenômenos devem ainda 
ser determinadas (LORETOet al., 2014). Além disso, numa série clínica, 20\% dos indivíduos com calvície de padrão masculino relataram disfunção sexual persistente, o que sugere a possibilidade de que a disfunção pode ser permanente. Esses eventos negativos e duradouros prejudicam a saúde sexual dos homens e provoca uma perda severa em termos de qualidade de vida (IRWIG, 2012).

Outros estudos indicam que a conversão de testosterona em DHT não é necessária para ereção peniana, visto que ela é o hormônio responsável por essa ação. Dessa maneira, a diminuição da DHT causada pela finasterida não dificultaria a ereção. Complementando esses estudos, ensaios clínicos não relataram diferença estatisticamente significativa entre os escores obtidos em doentes que receberam o placebo e finasterida (TOSTIet al., 2004; TOSTI et al., 2001). No entanto, esses estudos não levam em consideração a possibilidade de efeitos neuro-endócrinos.

\section{FINASTERIDA E FERTILIDADE}

Com relação à espermatogênese, concluiu-se, a partir de um estudo, que ela não foi adversamente afetada pela droga; no entanto, foram analisados apenas pacientes férteis excluindo os pacientes com a análise do sêmen de rastreio anormal, história de infertilidade, criptorquidia e varicocele (CHIBAet al., 2011). Diferentemente, em outra análise, foram relatados dois casos de pacientes inférteis com azoospermia ou oligospermia grave que mostraram melhorias significativas nas concentrações de esperma após a descontinuação de $1 \mathrm{mg}$ de finasterida, e sugeriu-se que a diminuição dos níveis de DHT locais podem desempenhar um papel na espermatogênese em homens com subfertilidade preexistente (LIU et al., 2008). Outros estudos relacionados a parâmetros seminais mostraram redução na contagem de espermatozoides no inicio do tratamento com normalização desse parâmetro depois de 52 semanas. Isso indica que a testosterona por si só, pode ser suficiente para manter a espermatogênese (AMORYet al., 2013; ANITHA et al., 2009).

\section{ASPECTOS PSICOLÓGICOS RELACIONADOS À PRESCRIÇÃO DA FINASTERIDA}

É necessário avaliar o risco e benefício do tratamento da alopéciaandrogenética com finasterida. A AAG é uma doença que pode trazer consequências mais relevantes que os efeitos adversos do seu tratamento. Alguns pacientes tendem a avaliar sua perda de cabelo como mais grave do que os dermatologistas. Consequentemente, a compreensão da preocupação psicossocial e qualidade de vida de pacientes com AAG tornou-se um assunto de grande preocupação(HANet al., 2012). Por 
isso, a abordagem médica das questões psicossociais e de qualidade de vida tem sido sugerida no tratamento de doentes com alopecia (GANZERet al., 2014).

Além disso, um estudo que avaliou as modificações na qualidade de vida dos pacientes antes e depois do tratamento concluiu queo uso de finasterida oral melhora a qualidade de vida desses pacientes (YAMAZAKIet al., 2011).

Ademais, Mondaini e cols. descreveram o fenômeno "nocebo", em que um efeito adverso não é resultante da ação farmacológica de uma droga, mas sim do conhecimento do paciente acerca desse efeito adverso. Testaram essa hipótese em 120 pacientes em uso de finasterida para tratamento de hiperplasia prostática benigna, que, de forma randomizada, foram atribuídos a grupos que eram ou não informados sobre os efeitos adversos da medicação. Após seis e 12 meses, o grupo informado reportou uma incidência significativamente mais alta $(43 \%)$ de efeitos colaterais sexuais do que o grupo não informado (15\%). Conclui-se, com isso, que os efeitos colaterais sexuais podem possuir causa psicológica (MONDANIet al., 2007).

\section{CONCLUSÃO}

Os médicos que tratam a perda de cabelo com padrão masculino devem discutir os potenciais efeitos adversos dos medicamentos com os seus pacientes, uma vez que, em alguns indivíduos, estes efeitos secundários são persistentes no que diz respeito à função sexual e à carga emocional, incluindo diminuição da qualidade de vida.

Os possíveis impactos dos possíveis efeitos adversos relacionados ao uso da finasterida sobre a saúde do homem permanece no foco de estudos e controvérsias científicas e abrange questões que vão desde efeitos há muito tempo relatados até mecanismos que somente seriam explicados levando-se em consideração as fronteiras da neurociência e fisiologia. Por outro lado, os defensores dessa terapêutica advogam que a persistência dos efeitos colaterais parece ser um evento raro, eque ainda deve ser determinado se representam uma relação causal verdadeira, ou se eles são simplesmente uma coincidência e relacionados a outros fatores como a alta incidência de disfunção sexual na população. Ademais, alegam que milhões de pacientes se beneficiam do uso da finasterida sem apresentarem efeitos colaterais. Logo, faz-se necessária a realização de mais estudos para que informações precisas possam ser dadas aos pacientes, que lhes permitam fazer 
escolhas em relação ao uso deste

medicamento.

Portanto, sugere-se avaliar cada paciente individualmente, visto que cada um possui uma visão da doença que porta e poderá apresentar um perfil de resposta diferente ao tratamento. Dessa maneira, o médico e a equipe de saúde podem, juntamente com seu paciente, chegar à melhor conduta.

Também é válido ressaltar que o encontro entre médico e paciente proporcionado pela queixa de calvície ou queda capilar, consiste em uma importante oportunidade para se abordar outras esferas da saúde masculina, como a existência de comorbidades não diagnosticadas, combate a violência ou comportamentos de risco e orientações sobre sexualidade e saúde reprodutiva.

\section{REFERÊNCIAS}

AMORY, John K., et.al. The Effect of $5 \alpha$ Reductase Inhibition with Dutasteride and Finasteride on Semen Parameters and Serum Hormones in Healthy Men. The Journal of Clinical Endocrinology \& Metabolism. Carolina do Norte. 02 Jul.2013, vol.92,nº5,p.2006-2203.

ANITHA, B.; INAMADAR, Arun C.; RAGUNATHA, S. Finasteride - its impact on sexual function and prostate cancer.Journal of cutaneous and aesthetic surgery.Nova Delhi.Jan- Jun. 2009, v. 2, n.1, p. 12-16.
BELKNAP, Steven M., et. al. Adverse Event Reporting in Clinical Trials of Finasteride for Androgenic Alopecia: a meta-analysis. The Journal of the American Medical Association. Boston, 01 April 2015. vol. 36.

BRENNER, Fabiane Mulinari; SOARES, IvyFaigle. AlopéciaAndrogenética Masculina: Uma Atualização. Revista de Ciências Médicas. Campinas. Mai-Jun. 2009, v.18, n. 3, p. 153-161.

CARRARA, Sérgio; RUSSO, Jane A.; FARO, Livi. A política de atenção à saúde do homem no Brasil: os paradoxos da medicalização do corpo masculino. Revista de Saúde Coletiva, Rio de Janeiro, v.19, n.3, p.659-678, setembro. 2009.

CHIBA, Koji, et.al. Finasteride-associated male infertility.Fertility and Sterility. April 2011. v. 95, n. 5, p. 1786.e9-1786.e11.

GANZER, C.A., JACOBS,A.R.,IQBAL,F. Persistent sexual, emotional, and cognitive impairment post-finasteride: a survey of men reporting symptoms. American Journal of Men's Health. New York. 13 Jun. 2014,vol.9, $\mathrm{n}^{\circ} 3, \mathrm{p} .222-228$.

HAJHEYDARI, Zohreh, et. al. Comparing the therapeutic effects of finasteride gel and tablet in treatment of the androgenetic alopecia. Indian Journal of dermatology, venereology and leprology, Sari, 2009. v.75, p. 47-51

HAN, Sung-Hyub, et.al. Quality of Life Assessment in Male Patients with Androgenetic Alopecia: Result of a Prospective, Multicenter Study. Annals of Dermatology. Seoul. Aug. 2012,vol.24,n³,p.311-318.

IRWIG, Michael S. Persistent Sexual Side Effects of Finasteride: Could They be Permanent? The Journal of Sexual Medicine.Washington, 2012.V.9, p. 29272932.

IRWIG, Michael S.; KOLUKULA, Swapna.Persistent Sexual Side Effects of Finasteride for Male Pattern Hair Loss. The 
Journal of Sexual Medicine. San Diego, Jun 2011. v.8, n.6, p.1747-1753.

KAUFMAN, Keith D. Androgens and Alopecia.Molecular and Cellular Endocrinology.Rahway, v.198, n.1-2, p.8995.Dec. 2002.

KUNELIUS, Pekka, et.al. The Effects of Transdermal Dihydrotestosterone in the Aging Male: A Prospective, Randomized, Double Blind Study. The Journal of Clinical Endocrinology and Metabolism. Washington. 2 Jul. 2013. v.87, n.4.

LEE, J., CHANG, C. Recent advances in androgen receptor action. Cellular and Molecular Life Sciences CMLS, Switzerland, v.60, n.8, p. 1613-1622, August 2003.

LIU, Kimberly E.; BINSALEH, Saleh; LO, Kirk C.; JARVI, Keith.Propecia-induced spermatogenic failure: a report of two cases. Fertility and Sterility.Sept.2008, vol.90, n⿳3 3, p.849.e17-849.e19.

LORETO, Carla Di, et. al. Immunohistochemical Evaluation of Androgen Receptor and Nerve Structure Density in Human Prepuce from Patients with Persistent Sexual Side Effects after Finasteride Use for Androgenetic Alopecia. Plos one.San Francisco, 24 jun. 2014.

MAHMOUD, Rand, et al. Sex hormones and adult hippocampal neurogenesis: Regulation, implications, and potential mechanisms, Frontiers in Neuroendocrinology, (2016), doi: http://dx.doi.org/10.1016/j.yfrne.2016.03.002 MELCANGI, Roberto C., et.al. Neuroactive Steroid Levels are Modified in Cerebrospinal Fluid and Plasma of Post-Finasteride Patients Showing Persistent Sexual Side Effects and Anxious/Depressive Symptomatology. The journal of Sexual Medicine. Washington. 24 jul.2013, vol.10, nº10, p.2598-2603.

MONDANI, Nicola, et.al. Finasteride $5 \mathrm{mg}$ and Sexual Side Effects: How Many of these are Related to a Nocebo Phenomenon? The Journal of Sexual Medicine. Washington. Nov. 2007,vol.4,nº,p.1708-1712.
MOTOFEI, Ion G., et. al. A pilot study on the sexual side effects of finasteride as related to hand preference for men undergoing treatment of male pattern baldness. British Journal of Urology. Oxford, Apr 2013. V.111, n.4b, p. E221-E226.

MYSORE, Venkataram. Finasteride and sexual side effects.Indian Dermatology Online Journal. Bangalore, Jan-Apr. 2012. v.3, n.1, p.62-65.

PRICE, Vera H.; MENEFEE, Emory; STRAUSS, Paul C. Changes in hair weight and hair count in men with androgenetic alopecia, after application of 5\% and $2 \%$ topical minoxidil, placebo, or no treatment. Journal of the American Academy of Dermatology. San Francisco, 24 Jun. 1999. V.41, n.5, p. 717-721.

ROMER, B., Pfeiffer, N., Lewicka, S., BenAbdallah, N., Vogt, M.A., Deuschle, M., Vollmayr, B., Gass, P., 2010. Finasteride treatment inhibits adult hippocampal neurogenesis in male mice.

Pharmacopsychiatry 43, 174-178. SINGH, Meena K.; AVRAM, Marc. Persistent Sexual Dysfunction and Depression in Finasteride Users for Male Pattern Hair Loss. The Journal of Clinical and Aesthetic Dermatology. New York, Dec 2014. V.7, n.12, p. 51-55.

STOUGH, Dow, et.al. Psychological Effect, Pathophysiology, and Management of Androgenetic Alopecia in Men.Mayo Clinic Proceedings, Florida, v.80, n.10, p.13161322, Oct, 2005.

TOSTI, A.; PIRACCINI, B. M.; SOLI, M. Evaluation of sexual function in subjects taking finasteride for the treatment of androgenetic alopecia. Journal of the European Academy of Dermatology and Venereology.Munique.Sept.2001,vol.15,nº5,p .418-421.

TOSTI, Antonella, et.al. Evaluation of Sexual Function With an International Index of Erectile Function in Subjects Taking Finasteride for Androgenetic Alopecia. The 
Journal of the American Medical Association. Boston. 1 Jul.2004,vol.140,nº 7,p.857-858.

TRAISH, Abdulmaged M., et al. Adverse Side Effects of $5 \alpha$-Reductase Inhibitors Therapy: Persistent Diminished Libido and Erectile Dysfunction and Depression in a Subset of Patient. The Journal of Sexual Medicine. San Diego, Mar 2011. v.8, n.3, p.872-884.

WHITING, David A, et.al. Efficacy and tolerability of finasteride $1 \mathrm{mg}$ in men aged 41 to 60 years with male pattern hair loss.
European Journal of Dermatology. Dallas, Mar-Apr 2003. v.13, n.2, p.150-160.

WINIARSKA, A., et.al. Effect of 5 5 Dihydrotestosterone and Testosterone on Apoptosis in Human Dermal Papilla Cells.Journal of Pharmacological and Biophysical Research. Berlin, nov. 2006. v.19, n.6. p. 311-321.

YAMAZAKI, Masashi, et. al. Oral finasteride improved the quality oh life androgenetic alopecia patients. The Journal of Dermatology.Malden. Aug. 2011, v. 38, n. 8, p. 773-777. 